\title{
Efeito de banhos terapêuticos com formalina sobre indicadores de estresse em tambaqui
}

\author{
Lucelle Dantas de Araújo ${ }^{(1)}$, Edsandra Campos Chagas ${ }^{(2)}$, Levy de Carvalho Gomes ${ }^{(2)}$ e Franmir Rodrigues Brandão(3) \\ (1)Escola Superior Batista do Amazonas (ESBAM), Rua Rio Javari, 278, CEP 69057-510 Manaus, AM. Bolsista do PIBIC/CNPq. \\ E-mail: lucelle@cpaa.embrapa.br (2)Embrapa Amazônia Ocidental, Caixa Postal 319, CEP 69011-970 Manaus, AM. \\ E-mail: edsandra@cpaa.embrapa.br, levy@cpaa.embrapa.br (3)Centro Universitário Nilton Lins, Av. Prof. Nilton Lins, 3259, CEP 69058-040 Manaus, \\ AM. Bolsista do PIBIC/CNPq. E-mail: franmir@cpaa.embrapa.br
}

Resumo - A formalina tem sido usada no controle de doenças parasitárias, porém existem poucas informações sobre o efeito secundário desse produto sobre os peixes. O objetivo deste trabalho foi avaliar o efeito de concentrações terapêuticas de formalina na homeostase de tambaqui (Colossoma macropomum Cuvier 1818) por meio de índices fisiológicos. Os banhos terapêuticos foram realizados em tanques de $100 \mathrm{~L}$, com soluções de formalina a 0,100,150, 200 e 250 mg/L, em tempos de exposição de 0, 30, 60 e 120 minutos; a recuperação dos animais em tanques, sem adição de formalina à água, foi avaliada após 24 horas. Foi realizada a coleta de sangue da veia caudal dos peixes para determinação de glicose, cloretos, sódio, potássio e cálcio. Os peixes não exibiram sinais de estresse na concentração de $100 \mathrm{mg} / \mathrm{L}$ de formalina em todos os tempos de exposição. Contudo, banhos de 200 e $250 \mathrm{mg} / \mathrm{L}$ causaram aumentos significativos nos níveis de glicose após 30 minutos de exposição. Os resultados sugerem que a formalina pode ser usada nas concentrações de 100 e $150 \mathrm{mg} / \mathrm{L}$ em banhos de 30, 60 e 120 minutos e nas concentrações de 200 e $250 \mathrm{mg} / \mathrm{L}$ em banhos de até 30 minutos sem comprometer a homeostase do tambaqui.

Termos para indexação: Colossoma macropomum, homeostase, piscicultura.

\section{Effect of formalin therapeutic bath on stress indicators in tambaqui}

\begin{abstract}
The formalin has been used to control fish disease, however there are little information on secondary effect of this chemical on fish. The objective of this work was to evaluate the effect of formalin therapeutic concentrations on tambaqui (Colossoma macropomum Cuvier 1818) homeostasis by physiological parameters. Therapeutic baths were carried out in tanks of $100 \mathrm{~L}$ with formalin solution at 0, 100, 150, 200 and $250 \mathrm{mg} / \mathrm{L}$ and exposures of $0,30,60$ and 120 minutes; recovery of the animals in water without formalin solution was evaluated after 24 hours. Blood was withdrawn from caudal vein to evaluate glucose, chlorides, sodium, potassium and calcium. Fish did not present stress signs in the formalin concentration of $100 \mathrm{mg} / \mathrm{L}$ in all exposure times. Although, baths of 200 and $250 \mathrm{mg} / \mathrm{L}$ promote significant increase in glucose levels after 30 minutes exposure. Formalin might be used in bath solutions of 100 and $150 \mathrm{mg} / \mathrm{L}$ for 30, 60 and 120 minutes and in baths of 200 and $250 \mathrm{mg} / \mathrm{L}$ up to 30 minutes without compromising tambaqui homeostasis.
\end{abstract}

Index terms: Colossoma macropomum, homeostasis, fish culture.

\section{Introdução}

A aqüicultura vem crescendo, nos últimos anos, numa taxa de 30\% ao ano no Brasil (FAO, 1999), e uma das espécies de peixes nativas com excelente potencial para o cultivo é o tambaqui, Colossoma macropomum Cuvier 1818. Esta espécie apresenta bom crescimento, hábito gregário, resistência a baixos níveis de oxigênio dissolvido e excelente utilização de alimentos (Saint-Paul, 1986; Val et al., 1998).

A intensificação da piscicultura pode conduzir ao aparecimento de doenças, mas se forem diagnosticadas cedo, mediante exames parasitológicos, estas podem ser facilmente tratadas (Pavanelli et al., 1998).

No tratamento das doenças em peixes, vários protocolos têm sido utilizados, como os banhos terapêuticos, o uso de rações medicadas e a aplicação de vacinas (Thune et al., 1997; Wise \& Johnson, 1998; Reed et al., 1999; Wise et al., 2000; Fouz et al., 2001). Em banhos terapêuticos, os produtos químicos mais utilizados no tratamento de doenças são a formalina, o permanganato de potássio e o cloreto de sódio, sal de cozinha (Kabata, 1985; Thatcher, 1991; Pavanelli et al., 1998, Griffin et al., 2002). 
A formalina é indicada para controlar infecções parasitárias externas, atuando sobre parasitas de brânquias, pele e nadadeiras, sendo efetiva contra a maioria dos protozoários e trematódeos monogenéticos (Kabata, 1985; Francis-Floyd, 1995; Pironet \& Jones, 2000; FajerÁvila et al., 2003). As concentrações utilizadas para banhos de curta duração estão entre 125 e 250 mg/L; em tratamentos de tempo indefinido estão entre $15 \mathrm{e}$ 25 mg/L (Schnick, 1973; Kabata, 1985; Ceccarelli et al., 1993; Li et al., 1996; Pironet \& Jones, 2000).

O emprego de formalina em banhos terapêuticos na aqüicultura já é liberado pelas agências de saúde dos Estados Unidos (Francis-Floyd, 1995; Fajer-Ávila et al., 2003). No Brasil, ainda não existe uma legislação específica liberando o uso deste produto no tratamento de doenças em peixes tropicais, uma vez que é pouco conhecido o efeito prejudicial do estresse que a formalina promove nos peixes (Kakuta et al., 1991).

O objetivo deste trabalho foi avaliar o efeito de concentrações terapêuticas de formalina na homeostase de tambaqui, por meio de indicadores fisiológicos de estresse.

\section{Material e Métodos}

Juvenis de tambaqui $(37,80 \pm 0,24 \mathrm{~g}$ e 13,48 $\pm 0,48 \mathrm{~cm})$ foram distribuídos em tanques de polietileno, com o volume fixo de $100 \mathrm{~L}$, segundo delineamento inteiramente casualizado dos tratamentos, que consistiram nas concentrações de formalina de $0,100,150,200$ e $250 \mathrm{mg} / \mathrm{L}$ com três repetições. A alimentação dos animais foi suspensa 24 horas antes do início dos testes e durante os mesmos, sendo os peixes aclimatados nos tanques experimentais por um período de 24 horas. Os parâmetros físico-químicos da água dos tanques foram monitorados, diariamente, durante a execução dos testes. $\mathrm{O} \mathrm{pH}$ foi de $6,81 \pm 0,05$, a temperatura, $26,93 \pm 0,23^{\circ} \mathrm{C}$, e o oxigênio dissolvido, 7,57 $\pm 0,09 \mathrm{mg} / \mathrm{L}$.

Antes da exposição (0 minutos) e após, 30, 60 e 120 minutos de exposição à formalina, foram retirados nove peixes de cada tratamento (três de cada repetição) para a coleta de sangue, visando à avaliação do estado de saúde dos peixes. Após anestesia com 100 mg/L de benzocaína (Gomes et al., 2001), procedeu-se à coleta de sangue mediante punção de vasos caudais com seringas heparinizadas; as amostras foram acondicionadas em gelo fundente, até o momento de uso. Após 120 minutos de exposição, a água dos tanques foi trocada de modo a remover toda a formalina presente no tanque, e os peixes permaneceram sob observação por
24 horas. Após este período de recuperação, foi feita outra coleta de sangue. Durante o período experimental, foi avaliada a sobrevivência dos peixes.

A glicose foi determinada pelo método da glicose oxidase e o cloreto plasmático, usando um kit comercial específico. Os níveis de $\mathrm{Na}^{+}$e $\mathrm{K}^{+}$foram determinados em fotômetro de chama e os níveis de $\mathrm{Ca}^{2+}$ estimados por espectrofotometria de absorção atômica.

As diferenças obtidas entre os tratamentos foram estabelecidas por análise de variância de dois fatores e as médias comparadas com o controle pelo teste de Dunnett, a 5\% de probabilidade (Zar, 1999).

\section{Resultados e Discussão}

No período anterior à exposição (0 minutos), não foi observada diferença nos níveis de glicose entre as concentrações de formalina (Tabela 1). Após 30, 60 e 120 minutos de exposição, houve aumento dos níveis de glicose nos tratamentos de 200 e $250 \mathrm{mg} / \mathrm{L}$. Foi observado aumento significativo nos níveis de glicose também no grupo de $150 \mathrm{mg} / \mathrm{L}$ de formalina nos tempos de exposição de 60 e 120 minutos.

A glicose plasmática, que desempenha importante papel no metabolismo dos peixes, é um dos indicadores mais utilizados, juntamente com o cortisol, para diagnosticar a ocorrência de estresse fisiológico (Wedemeyer et al., 1990). Os tambaquis não exibiram uma resposta de estresse após 120 minutos de exposição no tratamento de $100 \mathrm{mg} / \mathrm{L}$, bem como no período de recuperação de 24 horas. Embora tenha ocorrido uma alteração nos níveis de glicose na concentração de $150 \mathrm{mg} / \mathrm{L}$ de formalina nos tempos de 60 e 120 minutos, ainda assim os valores observados estão próximos dos valores normais para tambaquis cultivados em tanques escavados (Marcon et al., 1997) e em tanques-rede (Chagas et al., 2003). Em contraste, nas concentrações de formalina de 200 e $250 \mathrm{mg} / \mathrm{L}$ após 30, 60 e 120 minutos, pode-se caracterizar a elevação nos níveis de glicose como consequiência de uma situação de estresse. Aumentos na glicose sanguínea associados com as respostas fisiológicas a diferentes protocolos de estresse como exposição ao nitrito (Paula-Silva, 1999) e produtos de excreção (Andrade, 1999), têm sido observados em tambaquis.

Nos diferentes tratamentos com formalina, não foi observada qualquer alteração nos valores de cloretos, sódio e potássio plasmático de tambaqui no período anterior à exposição, bem como nos tempos de exposição de 30, 60 e 120 minutos e após 24 horas de recuperação (Tabela 1). De uma forma geral, os valores dos íons, 
acima citados, estão dentro dos níveis basais encontrados para tambaquis cultivados em tanques-rede (Chagas et al., 2003).

No período pré-exposição, não foram observadas diferenças nos níveis de cálcio plasmático entre os tratamentos (Tabela 1). Por sua vez, os valores de cálcio nos peixes dos tratamentos de 200 e $250 \mathrm{mg} / \mathrm{L}$ de formalina aumentaram em relação ao controle e ao tempo de préexposição, aos 60 e 120 minutos de exposição. Estes resultados evidenciam uma alteração na homeostase iônica do tambaqui como resultado da elevação desordenada do cálcio plasmático, que é transportado de dentro das células para o plasma, levando ao aumento da concentração plasmática deste íon (Baldisserotto, 2003). O equilíbrio nos níveis de cálcio deve ser mantido para o perfeito funcionamento das funções neuromusculares e manutenção da homeostase orgânica (Shiau \& Hwang, 1993; Björnsson et al., 1999).

Ensaios com juvenis de pacu (Piaractus mesopotamicus) tratados com uma solução de formalina

Tabela 1. Teor de glicose, cloretos, sódio, potássio e cálcio no plasma sanguíneo de juvenis de tambaqui após exposição à formalina, em diferentes períodos e concentrações, e após 24 horas de recuperação ${ }^{(1)}$.

\begin{tabular}{|c|c|c|c|c|c|}
\hline \multirow{2}{*}{$\begin{array}{c}\text { Tempo de } \\
\text { exposição } \\
\text { (min) }\end{array}$} & \multicolumn{5}{|c|}{ Formalina $(\mathrm{mg} / \mathrm{L})$} \\
\hline & 0 & 100 & 150 & 200 & 250 \\
\hline & \multicolumn{5}{|c|}{ Glicose (mg/dL) } \\
\hline 0 & $63,76 \pm 4,10$ & $65,12 \pm 4,29$ & $68,70 \pm 1,58$ & $69,04 \pm 5,70$ & $69,64 \pm 5,87$ \\
\hline 30 & $71,20 \pm 4,91$ & $65,62 \pm 2,90$ & $78,96 \pm 3,76$ & $89,57 \pm 7,59 \mathrm{ab}$ & $125,51 \pm 8,93 \mathrm{ab}$ \\
\hline 60 & $73,21 \pm 1,40$ & $73,40 \pm 2,17$ & $86,37 \pm 3,49 a$ & $103,65 \pm 2,26 \mathrm{ab}$ & $111,14 \pm 5,43 \mathrm{ab}$ \\
\hline 120 & $75,11 \pm 1,57$ & $72,08 \pm 4,67$ & $89,09 \pm 6,05 a$ & $97,17 \pm 4,41 \mathrm{ab}$ & $122,77 \pm 11,86 a b$ \\
\hline \multirow[t]{2}{*}{ Recuperação } & $72,74 \pm 6,02$ & $70,95 \pm 6,01$ & $79,45 \pm 1,68$ & $75,29 \pm 4,84 \mathrm{ab}$ & $73,40 \pm 2,74$ \\
\hline & \multicolumn{5}{|c|}{ Cloretos (mmol/L) } \\
\hline 0 & $133,84 \pm 12,08$ & $138,53 \pm 12,85$ & $137,41 \pm 12,09$ & $134,13 \pm 12,97$ & $131,13 \pm 15,36$ \\
\hline 30 & $130,03 \pm 11,85$ & $148,38 \pm 13,65$ & $144,76 \pm 16,95$ & $151,88 \pm 12,04$ & $154,98 \pm 13,48$ \\
\hline 60 & $139,05 \pm 13,99$ & $148,21 \pm 14,57$ & $155,34 \pm 13,95$ & $162,96 \pm 17,55$ & $148,18 \pm 16,52$ \\
\hline 120 & $130,14 \pm 9,97$ & $148,60 \pm 11,51$ & $163,92 \pm 16,69$ & $144,18 \pm 18,88$ & $159,44 \pm 16,93$ \\
\hline \multirow[t]{2}{*}{ Recuperação } & $132,13 \pm 5,67$ & $140,39 \pm 14,20$ & $146,85 \pm 13,28$ & $138,31 \pm 9,43$ & $139,50 \pm 9,01$ \\
\hline & \multicolumn{5}{|c|}{ Sódio (mEq/L) } \\
\hline 0 & $142,32 \pm 3,91$ & $138,27 \pm 6,33$ & $140,01 \pm 5,37$ & $137,11 \pm 8,80$ & $142,90 \pm 6,22$ \\
\hline 30 & $139,24 \pm 5,12$ & $152,74 \pm 13,12$ & $138,66 \pm 2,43$ & $146,37 \pm 2,92$ & $137,12 \pm 5,45$ \\
\hline 60 & $129,98 \pm 3,93$ & $143,29 \pm 4,40$ & $140,59 \pm 2,74$ & $140,97 \pm 2,10$ & $142,32 \pm 5,52$ \\
\hline 120 & $149,52 \pm 4,29$ & $135,96 \pm 3,41$ & $134,61 \pm 4,88$ & $137,11 \pm 3,47$ & $134,61 \pm 3,71$ \\
\hline \multirow[t]{2}{*}{ Recuperação } & $145,99 \pm 2,71$ & $141,74 \pm 1,92$ & $135,57 \pm 2,83$ & $149,46 \pm 2,09$ & $139,24 \pm 0,99$ \\
\hline & \multicolumn{5}{|c|}{ Potássio (mEq/L) } \\
\hline 0 & $6,92 \pm 0,33$ & $6,98 \pm 0,20$ & $6,64 \pm 0,37$ & $6,28 \pm 0,31$ & $6,71 \pm 0,29$ \\
\hline 30 & $6,71 \pm 0,15$ & $6,97 \pm 0,23$ & $6,99 \pm 0,24$ & $6,96 \pm 0,28$ & $7,41 \pm 0,28$ \\
\hline 60 & $6,92 \pm 0,28$ & $6,81 \pm 0,14$ & $6,36 \pm 0,50$ & $6,36 \pm 0,28$ & $7,49 \pm 0,29$ \\
\hline 120 & $6,90 \pm 0,28$ & $6,81 \pm 0,29$ & $5,90 \pm 0,23$ & $7,15 \pm 0,38$ & $7,38 \pm 0,37$ \\
\hline \multirow[t]{2}{*}{ Recuperação } & $6,92 \pm 0,28$ & $6,90 \pm 0,28$ & $6,92 \pm 0,50$ & $6,98 \pm 0,26$ & $6,70 \pm 0,42$ \\
\hline & \multicolumn{5}{|c|}{ Cálcio (mEq/L) } \\
\hline 0 & $6,25 \pm 0,25$ & $6,11 \pm 0,24$ & $6,40 \pm 0,22$ & $6,39 \pm 0,19$ & $6,92 \pm 0,28$ \\
\hline 30 & $6,20 \pm 0,17$ & $6,66 \pm 0,22$ & $7,79 \pm 0,37$ & $7,06 \pm 0,24$ & $6,88 \pm 0,53$ \\
\hline 60 & $6,43 \pm 0,28$ & $6,99 \pm 0,21$ & $8,35 \pm 0,68$ & $9,12 \pm 1,08 \mathrm{ab}$ & $10,09 \pm 1,67 \mathrm{ab}$ \\
\hline 120 & $6,53 \pm 0,33$ & $7,16 \pm 0,19$ & $8,49 \pm 0,78$ & $9,56 \pm 1,17 \mathrm{ab}$ & $12,01 \pm 1,78 \mathrm{ab}$ \\
\hline Recuperação & $6,19 \pm 0,27$ & $6,83 \pm 0,23$ & $6,33 \pm 0,21$ & $6,32 \pm 0,18$ & $6,45 \pm 0,29$ \\
\hline
\end{tabular}

(1)Nas colunas, médias ( \pm erro-padrão) seguidas pela letra a apresentam diferença em relação à média do tratamento pré-exposição (tempo zero), e nas linhas, médias seguidas pela letra b diferem da média do tratamento controle de formalina $(0 \mathrm{mg} / \mathrm{L})$ a $5 \%$ de probabilidade, pelo teste de Dunnett. 
de $250 \mathrm{mg} / \mathrm{L}$, em banhos de curta duração (30 a 60 minutos), mostraram grande efetividade deste produto contra Tricodina sp. e Linguactyloides sp. (Cecarelli et al., 1993). Porém, as concentrações utilizadas no tratamento de alguns parasitas muitas vezes são próximas da concentração letal para a espécie alvo, devendo-se avaliar o efeito fisiológico de tais tratamentos. Em relação ao tambaqui, pôde-se observar, no presente trabalho, que na concentração de $250 \mathrm{mg} / \mathrm{L}$, geralmente recomendada no controle de monogenóides, o tempo máximo de exposição ao banho terapêutico deve ser de 30 minutos, pois após este período a homeostasia do tambaqui pode ser comprometida. Banhos de longa duração, acima de 120 minutos, não são tão efetivos no controle de parasitas, uma vez que a formalina é rapidamente consumida por átomos que ligam moléculas de partículas gerais e proteínas solúveis dentro da coluna da água (Williams \& Lightner, 1988).

\section{Conclusão}

A formalina pode ser empregada no tratamento de parasitas do tambaqui nas concentrações de $100 \mathrm{e}$ $150 \mathrm{mg} / \mathrm{L}$ em banhos de 30, 60 e 120 minutos e nas concentrações de 200 e $250 \mathrm{mg} / \mathrm{L}$ em banhos de até 30 minutos sem comprometer a homeostasia do tambaqui.

\section{Agradecimentos}

Ao Sr. José Pereira de Souza, pelo auxílio na condução dos experimentos no campo; à Embrapa Amazônia Ocidental, pelas análises nos laboratórios de Biotecnologia Vegetal e de Solos e Plantas; ao FINAM/ BASA, pelo apoio financeiro.

\section{Referências}

ANDRADE, I.P. Aspectos hematológicos, bioquímicos e microbiológicos de tambaqui, Colossoma macropomum (Cuvier, 1818): efeitos da carência alimentar, dos produtos de excreção e da infecção por microorganismos. 1999. 83p. Dissertação (Mestrado) Instituto Nacional de Pesquisas da Amazônia/Fundação Universidade do Amazonas, Manaus.

BALDISSEROTTO, B. Osmoregulatory adaptations of freshwater teleosts. In: VAL, A.L.; KAPOOR, B.G. (Ed.). Fish adaptations. Enfield: Science Publishers, 2003. p.179-202.

BJÖRNSSON, B.T.; PERSSON, P.; LARSSON, D.; JOHANNSSON, S.H.; SUNDELL, K. Calcium balance in teleost fish and endocrine control mechanisms. In: DANKS, J.; DACKE, C.; FLIK, G.; GAY, C. (Ed.). Calcium metabolism. Bristol: BioScientifica, 1999. p.1-10.

CECCARELLI, P.S.; ALCÂNTARA-ROCHA, R.C.G.; MELO, J.S.C. de. Efeitos do formaldeído sobre a Trichodina sp. e
Linguadactyloides sp. em alevinos de pacu, Piaractus mesopatamicus Holmberg, 1887. Boletim Técnico do CEPTA, v.6, p.23-30, 1993.

CHAGAS, E.C.; LOURENÇO, J.N.P.; GOMES, L.C.; VAL, A.L. Desempenho e estado de saúde de tambaquis cultivados em tanquesrede sob diferentes densidades de estocagem. In: SIMPÓSIO BRASILEIRO DE AQÜICULTURA, 12., 2002, Goiânia. Anais. Jaboticabal: Aquabio, 2003. p.83-93.

FAJER-ÁVILA, E.J.; PARRA, I.A.; AGUILAR-ZARATE, G.; CONTRERAS-ARCE， R.; ZALDÍVAR-RAMÍREZ， J.; BETANCOURT-LOZANO, M. Toxicity of formalin to bullseye puffer fish (Sphoeroides annulatus Jenyns, 1843) and its effectiveness to control ectoparasites. Aquaculture, v.223, p.41-50, 2003.

FAO (Roma, Itália). The state of world fisheries and aquaculture 1998. Roma, 1999. 112p.

FOUZ, B.; ESTEVE-GASSENT, M.D.; BARRERA, R.; LARSEN, J.L.; NIELSEN, M.E.; AMARO, C. Field testing of a vaccine against eel diseases caused by Vibrio vulnificus. Diseases of Aquatic Organisms, v.45, p.183-189, 2001.

FRANCIS-FLOYD, R. The use of salt in aquaculture. Gainesville: University of Florida, 1995. 6p. (Fact Sheet, 86).

GOMES, L.C.; CHIPPARI-GOMES, A.R.; LOPES, N.P.; ROUBACH, R.; ARAÚJO-LIMA, C.A.R.M. Efficacy of benzocaine as an anesthetic in juvenile tambaqui, Colossoma macropomum. Journal of the World Aquaculture Society, v.32, p.426-431, 2001.

GRIFFIN, B.R.; DAVIS, K.B.; DARWISH, A.; STRAUS, D.L. Effect of exposure to potassium permanganate on stress indicators in channel catfish Ictalurus punctatus. Journal of the World Aquaculture Society, v.33, p.1-9, 2002.

KABATA, Z. Parasites and diseases of fish cultured in the tropics. London: Taylor \& Francis, 1985. 318p.

KAKUTA, I.; NAMBA, K.; UEMATSU, K.; MURACHI, S. Physiological response of the fish, Cyprinus carpio, to formalin exposure -1 : effects of formalin on urine flow, heart rate, respiration. Comparative Biochemistry and Physiology, v.100C, p.405-411, 1991.

LI, M.H.; WISE, D.J.; ROBINSON, E.H. Chemical prevention and treatment of winter Saprolegniosis ("Winter kill") in channel catfish Ictalurus punctatus. Journal of the World Aquaculture Society, v.27, p.1-6, 1996.

MARCON, J.L.; OLIVEIRA, M.I.; SANTOS, A.C. Parâmetros do sangue de Colossoma macropomum cultivado na região de Manaus: valores de referência para diagnóstico da saúde de animais destinados ao cultivo: relatório final. Manaus: UFAM; CNPq, 1997. 37p.

PAULA-SILVA, M.N. Influência do nitrito sobre aspectos fisiológicos do tambaqui, Colossoma macropomum (Cuvier, 1818). 1999. 61p. Dissertação (Mestrado) - Instituto Nacional de Pesquisas da Amazônia/Fundação Universidade do Amazonas, Manaus.

PAVANELLI, G.C.; EIRAS, J.C.; TAKEMOTO, R.M. Doenças de peixes: profilaxia, diagnóstico e tratamento. Maringá: Ed. da Universidade Estadual de Maringá, 1998. 264p.

PIRONET, F.N.; JONES, J.B. Treatments for ectoparasites and diseases in captive Western Australian dhufish. Aquaculture International, n.81, p.349-361, 2000.

REED, P.; FRANCIS-FLOYD, R.; KLINGERR, R. Monogenean trematodes. Gainesville: University of Florida, 1999. 5p. (Fact Sheet, 28). 
SAINT-PAUL, U. Potential for aquaculture of South American freshwater fishes: a review. Aquaculture, v.54, p.205-240, 1986.

SCHNICK, R.A. Formalin as a therapeutant in fish culture. Washington: United States Fish and Wildlife Service, 1973. 26p.

SHIAU, S.Y.; HWANG, J.Y. Vitamin D requirements of juvenile hybrid tilapia Oreochromis niloticus x $O$. aureus. Bulletin of the Japanese Society for Scientific Fisheries, v.59, p.553-558, 1993.

THATCHER, V.E. Parasites of Amazon fishes. Amazoniana, v.9, p.263-572, 1991.

THUNE, R.L.; COLLINS, L.A.; PEÑA, M.P. A comparison of immersion, immersion/oral combination and injection methods for the vaccination of channel catfish Ictalurus punctatus against Edwardsiella ictaluri. Journal of the World Aquaculture Society, v.28, p.193-201, 1997.

VAL, A.L.; SILVA, M.N.P.; ALMEIDA-VAL, V.M.F. Hypoxia adaptation in fish of the Amazon: a never-ending task. South African Journal of Zoology, v.33, p.107-114, 1998.

WEDEMEYER, G.A.; BARTON, B.A.; McLEAVY, D.J. Stress and acclimation. In: SCHRECK, C.B.; MOYLE, P.B. (Ed.). Methods for fish biology. Bethesda: American Fisheries Society, 1990. p.451-490.

WILLIAMS, R.R.; LIGHTNER, D.V. Formalin loss in seawater associated with varying levels of organic detritus. Journal of the World Aquaculture Society, v.19, p.163-168, 1988.

WISE, D.J.; JOHNSON, M.R. Effect of feeding frequency and Romet-Medicated feed on survival, antibody response, and weight gain of fingerling channel catfish Ictalurus punctatus after natural exposure to Edwardsiella ictaluri. Journal of the World Aquaculture Society, v.29, p.169-175, 1998.

WISE, D.J.; KLESIUS, P.H.; SHOEMAKER, C.A.; WOLTERS, W.R. Vaccination of mixed and full-sib families of channel catfish Ictalus punctatus against enteric septicemia of catfish with a live attenuated Edwardsiella ictaluri isolate (RE-33). Journal of the World Aquaculture Society, v.31, p.206-212, 2000.

ZAR, J.H. Biostatiscal analysis. $4^{\text {th }}$ ed. Upper Saddle River: Prentice Hall, 1999. 663p.

Recebido em 15 de agosto de 2003 e aprovado em 11 de dezembro de 2003 